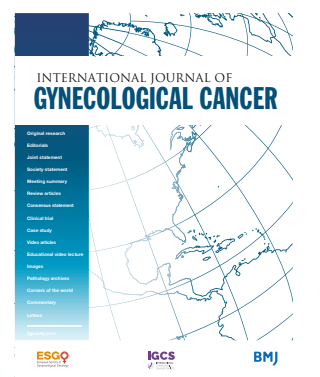

\title{
Ultrasound, macroscopic and histological features of malignant ovarian tumors. Metastatic tumors to the ovary: ovarian metastases from biliary tract and ovarian metastases from colon cancer
}

Francesca Ciccarone, Claudia Codecà, Valeria Versace, Francesca Moro

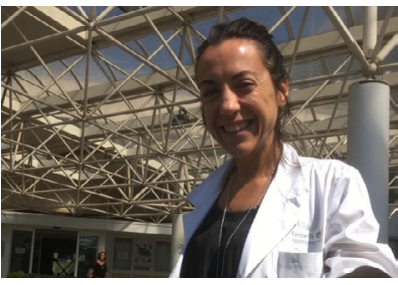

\section{Francesca Moro}

'Dipartimento Scienze della Salute della Donna, del Bambino e di Sanità Pubblica, Fondazione Policlinico Universitario Agostino Gemelli, IRCCS, Rome, Italy

Biography: Dr Francesca Moro is a gynecologist of Fondazione Policlinico Universitario Agostino Gemelli, IRCCS, in Rome. She is particularly involved in clinical research in ultrasound and gynecologic oncology.

For numbered affiliations see end of article.

Correspondence to Dr Francesca Moro, Dipartimento Scienze della Salute della Donna, del Bambino e di Sanità Pubblica, Fondazione Policlinico Universitario Agostino Gemelli, IRCCS, Rome, Italy; morofrancy@gmail.com

Accepted 19 January 2021

\section{INTERNATONAL OURNAL OF GYNECOLOGICAL CANCER}

\section{CLINICAL INFORMATION}

The patient was referred to our center for bilateral solid adnexal masses and free fluid in the pouch of Douglas that were incidentally detected during a routine transvaginal ultrasound examination performed at another hospital.

The patient reported weight loss and se ovels of oncological markers were:

CA 125249 U/ml, CA 19.9117 U/ml, C, $19 \mathrm{U} / \mathrm{ml}$, CEA $57 \mathrm{ng} / \mathrm{ml}$. (c) IGCS and ESGO 2021. № commercial re-use. See rights and permissions. Published by BMJ.

To cite: Ciccarone $F$, Codecà $\mathrm{C}$, Versace $\mathrm{V}$, et al. Int J Gynecol Cancer 2021;31:1388-1390.

\section{@IJGConline}

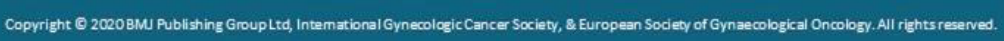

Video 1 Two cases of ovarian metastases. The first video shows clinical, ultrasound, macroscopic and histological features of a patient with ovarian metastasis from biliary tract. The second video presents clinical, ultrasound, macroscopic and histological features of a patient with ovarian metastases from colon cancer. 


\section{SUMMARY}

The objective of this video is to present two cases of ovarian metastases, examined at the Gynecologic Oncology Unit of the Fondazione Policlinico Universitario Agostino Gemelli, IRCCS, in Rome, Italy.

The first case is a 79-year-old patient with no family history of cancer and a past medical history of cholangiocarcinoma and left oophorectomy for ovarian metastasis. She was referred to our center for omental cake and a right adnexal mass detected at CT scan during oncological follow-up. The patient was asymptomatic and serum levels of oncological markers were: CA 19.9 155U/ $\mathrm{mL}$ (reference range $0-37 \mathrm{U} / \mathrm{mL}$ ), CEA $1.4 \mathrm{ng} / \mathrm{mL}$ (reference range $0-5 \mathrm{ng} / \mathrm{mL}$ ). Transvaginal ultrasound examination performed at our center showed a right multilocular solid tumor ( $>10$ locules) of $96 \times 113 \times 94 \mathrm{~mm}$, with low-level content and irregular internal walls with a solid component of $38 \times 22 \times 23 \mathrm{~mm}$ in size. ${ }^{1}$ The mass showed rich vascularization at color Doppler examination. IOTA $\mathrm{ADNEX}^{2}$ showed an increased risk of malignancy, with highest relative risk either for stage II-IV ovarian cancer or for metastatic cancer to the adnexa (link to the IOTA ADNEX model calculator: https://www.iotagroup.org/sites/default/files/adnexmodel/IOTAADNEXmodel.html). Moreover, the tumor was classified as OvarianAdnexal Reporting and Data System (0-RADS) 5. ${ }^{3}$

At laparoscopy, the presence of the right ovarian mass was confirmed. Thus, a right salpingo-0ophorectomy and peritoneal biopsies were performed.

The macroscopic assessment confirmed the presence of a solid cystic neoformation of $14 \mathrm{~cm}$ in size and the absence of residual ovarian parenchyma. ${ }^{4}$ The final histology report described ovarian tissue infiltrated by adenocarcinoma with morphological and immunohistochemical aspects compatible with biliary tract carcinoma. ${ }^{5}$ Tubal tissue and peritoneal biopsies were negative for atypia.

The second case is a 69-year-old patient with no family history of cancer and a previous laparotomy performed for a myomectomy. She was referred to our center for bilateral solid adnexal masses and free fluid in the pouch of Douglas that were incidentally detected during a routine transvaginal ultrasound examination performed at another hospital. The patient reported weight loss and serum levels of oncological markers were: CA $125249 \mathrm{U} / \mathrm{mL}$ (reference range $0-35 \mathrm{U} / \mathrm{mL}$ ), CA $19.9117 \mathrm{U} / \mathrm{mL}$ (reference range $0-37 \mathrm{U}$ / $\mathrm{mL}$ ), CA $15.319 \mathrm{U} / \mathrm{mL}$ (reference range $0-32.5 \mathrm{U} / \mathrm{mL}$ ), CEA $57 \mathrm{ng} /$ $\mathrm{mL}$ (reference range $0-5 \mathrm{ng} / \mathrm{mL}$ ). Transvaginal ultrasound examination was performed at our center and it showed bilateral adnexal solid masses with inhomogeneous echostructure, irregular external walls, and no stripes. ${ }^{1}$ The right solid mass was $118 \times 78 \times 67 \mathrm{~mm}$ in size and the left solid mass was $74 \times 60 \times 64 \mathrm{~mm}$ in size. At color Doppler examination, the right ovarian mass showed moderate vascularization and presence of the lead vessel was also observed. ${ }^{6}$ We applied the IOTA ADNEX model ${ }^{2}$ to the target lesion-the right solid mass. IOTA ADNEX showed an increased risk of malignancy, with highest relative risk either for stage II-IV ovarian cancer or for metastatic cancer to the adnexa (link to the IOTA ADNEX model calculator: https://www.iotagroup.org/sites/default/files/adnexmodel/IOTA-ADNEXmodel.html). Moreover, the tumor was classified as 0 -RADS $5 .^{3}$

MRI showed bilateral adnexal solid masses of $11 \mathrm{~cm}$ (right mass) and $5.5 \mathrm{~cm}$ (left mass), respectively; ascites, abdominal and pelvic

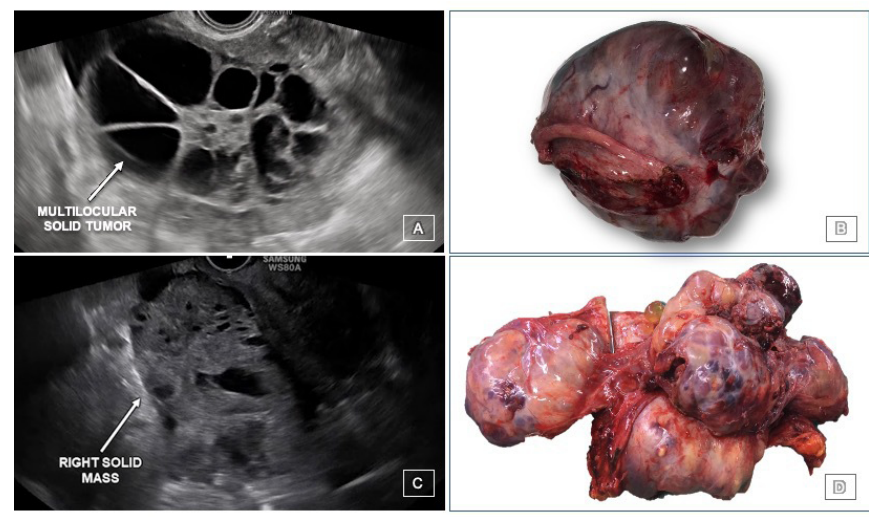

Figure 1 Ultrasound and macroscopic images of a 79-yearold patient with ovarian metastasis from biliary tract $(A, B)$ and a 69-year-old patient with ovarian metastasis from colon cancer (C, D).

lymphadenopathy, and nodules in both liver and right adrenal gland were present.

The patient underwent an exploratory laparoscopy, which confirmed the presence of bilateral adnexal masses with rectosigmoid compression. Multiple iliac and para-aortic lymphadenopathies were also visible. At frozen section, ovarian biopsies were positive for low-grade serous ovarian carcinoma. Therefore, the patient underwent a laparotomy and total hysterectomy, bilateral adnexectomy and biopsy of the superior mesenteric artery lymph node were performed.

At macroscopic examination, bilateral solid tumors were detected. ${ }^{4}$ The final histology report was positive for colon cancer ovarian metastases. $^{5}$

The patient underwent a colonoscopy, and a biopsy performed at the level of the recto-sigmoid junction was positive for adenocarcinoma.

A CT scan showed irregular recto-sigmoid thickening with perivisceral adipose tissue infiltration, abdominal and pelvic lymphadenopathy, peritoneal and liver metastases, as well as metastasis in the adrenal gland.

She was administered two courses of chemotherapy with CAPOX, which was then suspended for toxicity. She then underwent eight cycles of chemotherapy with FOLFOX and then four cycles of 5-fluorouracil and folic acid. Subsequently, because of progression of disease, the patient underwent a cycle of therapy with LONSURF, which was then suspended due to decay of general conditions.

\section{Author affiliations}

Dipartimento Scienze della Salute della Donna, del Bambino e di Sanità Pubblica, Fondazione Policlinico Universitario Agostino Gemelli, IRCCS, Rome, Italy

Contributors All the authors have made a significant contribution to this manuscript, have seen and approved the final manuscript, and have agreed to its submission to the International Journal of Gynecological Cancer.

Funding The authors have not declared a specific grant for this research from any funding agency in the public, commercial or not-for-profit sectors.

Competing interests None declared.

Patient consent for publication Not required.

Provenance and peer review Not commissioned; externally peer reviewed. 


\section{Educational video lecture}

\section{ORCID iD}

Francesca Moro http://orcid.org/0000-0002-5070-7245

\section{REFERENCES}

1 Testa AC, Ferrandina G, Timmerman D, et al. Imaging in gynecological disease (1): ultrasound features of metastases in the ovaries differ depending on the origin of the primary tumor. Ultrasound Obstet Gynecol 2007;29:505-11.

2 Van Calster B, Van Hoorde K, Valentin L, et al. Evaluating the risk of ovarian cancer before surgery using the ADNEX model to differentiate between benign, borderline, early and advanced stage invasive, and secondary metastatic tumours: prospective multicentre diagnostic study. BMJ 2014;349:g5920.
3 Andreotti RF, Timmerman D, Strachowski LM, et al. O-RADS us risk stratification and management system: a consensus guideline from the ACR Ovarian-Adnexal reporting and data system Committee. Radiology 2020;294:168-85.

4 Longacre TA, Wells M. Serous tumors. In: Kurman RJ, Carcangiu ML, Herrington CS, et al, eds. Who classification of tumours of female reproductive organs. Lyon, France: IARC Press, 2014: 15-24.

5 Seidman JD, Cho KR. Surface epithelial tumors of the ovary. In: Kurman RJ, Ellenson LH, Ronnet BM, eds. Blaustein's Pathology of the Female Genital Tract. 6th edn. Boston, MA: Springer, 2011: 679-784.

6 Testa AC, Mancari R, Di Legge A, et al. The 'lead vessel': a vascular ultrasound feature of metastasis in the ovaries. Ultrasound Obstet Gynecol 2008;31:218-21. 